\title{
The MPIA detector system for the LBT instruments LUCIFER and LINC-NIRVANA
}

\author{
Sebastiano Ligori ${ }^{a}$, Rainer Lenzen ${ }^{a}$, Holger Mandel $^{b}$, Bernhard Grimm $^{a}$, Ulrich Mall ${ }^{a}$ \\ ${ }^{a}$ Max-Planck-Institut für Astronomie, Königstuhl 17, 69117 Heidelberg, Germany; \\ ${ }^{b}$ Landessternwarte Heidelberg-Königstuhl, 69117 Heidelberg, Germany
}

\begin{abstract}
We describe the detector subsystem developed at MPIA to operate the Rockwell Hawaii-2 detectors used in the LUCIFER and LINC-NIRVANA instruments for the Large Binocular Telescope (LBT).

To fully exploit the capabilities of the LBT, the detector subsystem must meet, especially in the case of the low background applications foreseen for LUCIFER, very stringent requirements in terms of stability and read noise. A read-out electronics has been developed at MPIA, which is able to read the 32 outputs of the Hawaii- 2 detector, as well as the 4 reference signals available in this chip. The noise figure associated to the electronics alone is negligible with respect to the intrinsic read noise of the detector, while the cloking patterns and the value of the bias voltages applied to the chip are optimized in order to maximize the signal to noise ratio in the different operating modes.

We present the results of the tests performed with the LUCIFER science detector; in particular, we describe the main properties of the detector: read noise, dark current, linearity, and long term stability, and what are the read-out schemes foreseen for different observational modes.

We discuss also how the reference outputs can be used in order to correct for thermal drifts, and how effective those outputs are in removing higher frequency noise components.
\end{abstract}

Keywords: Interferometry, Electronics, Infrared Detectors

\section{INTRODUCTION}

The german contribution to the LBT instrumentation includes a couple of twin instruments for imaging and spectroscopy (LUCIFER I and II) to be installed at the bent Gregorian focus of both telescopes, as well as one of the beam-combining instrument, LINC-NIRVANA. Since all these Near Infrared (NIR) instruments will use the same kind of detector, namely the Rockwell Hawaii-2 HdCdTe $2048 \times 2048$ pxl ${ }^{2}$, the task to provide the read-out electronics for all these experiments has been assigned to the electronic and instrumentation groups of the Max-Planck-Institut für Astronomie (MPIA). This institute has a long tradition in realizing IR instrument, and one instrument equipped with a Hawaii-2 detector (OMEGA2000 ${ }^{1}$ ) is presently in the commissioning phase at the Calar Alto Observatory.

In the following we will describe the architecture of the MPIA read-out system, and its main characteristics. The requirements set by the different instruments will be discussed, and the actual performances of one such system, which should equip LUCIFER I and which is currently in the test phase, will be described and compared to the requirements.

Finally, we will discuss the open problems, the status and the foreseen schedule for the different instruments.

\section{THE DETECTOR}

As already mentioned, the detector which will be used is a Hawaii-2. This detector can be read-out in 4 or 32 output modes. The arrangement of the pixels on the detector is shown in the following figure. In order to reduce as much as possible the frame time, we plan to use routinely the 32 output mode. The possibility of switching to 4 output mode is desirable for troubleshooting, and will be implemented later. In addition to the 32 output

Further author information: (Send correspondence to S.L.)

S.L.: E-mail: ligori@mpia.de, Telephone: 496221528329 


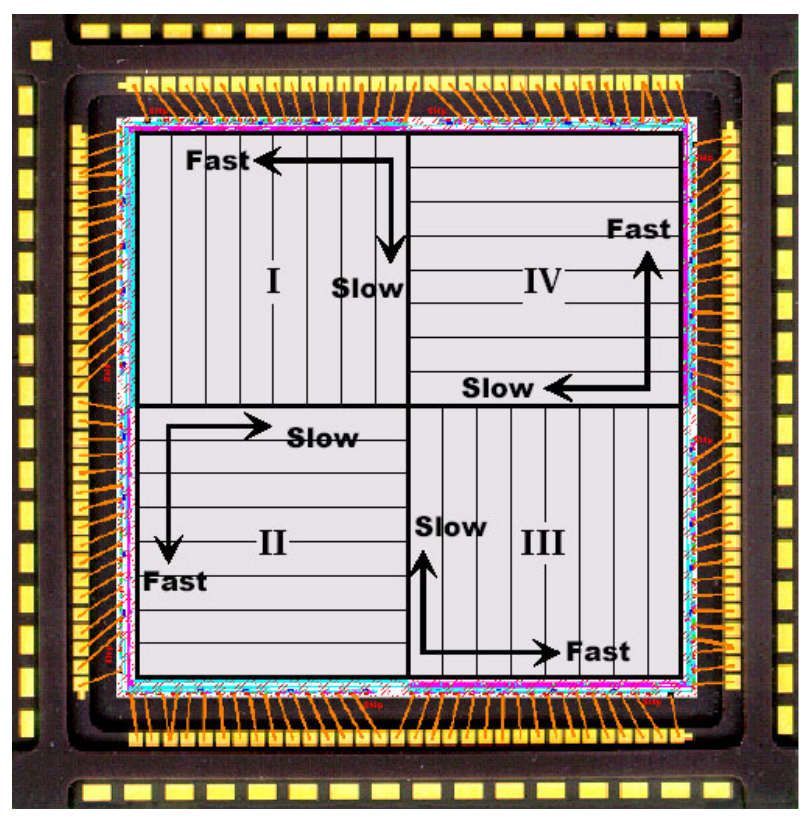

Figure 1. Detector Layout

channels, the Hawaii2 chip has four optional reference channels, which can be used to monitor low frequency drifts in the signal. The read-out electronics, must therefore be able to read-out a total of 36 channels. The frame time in 32 outputs is determined by the frame time, multiplied for the number of pixels in a $128 \times 1024$ channel $\left(1.31 \cdot 10^{5}\right)$.

Even though the maximum pixel clock frequency claimed by the manufacturer is $250 \mathrm{KHz}$, we found out that all the chips we tested have a much slower raise time, so that we adopted a pixel time of about $7.2 \mu \mathrm{s}$; with this clock speed, the total time needed for a single frame is $0.95 \mathrm{~s}$. check numbers. Due to the low read out speed, the rest of the signal chain is not particularly stressed. For instance, the data rate from the read-out electronics can be safely reduced to $20 \mathrm{MHz}$. As can be seen in the scope shot, the signal is sampled in the plateau after stabilization time, so that the associated noise component is very small.

\subsection{Read-out modes}

The read-modes presently implemented are basically the standard ones normally adopted in most of the NIR cameras:

- Reset-Read mode;

- Reset-Read-Read mode (correlated double sampling, CDS);

- Line interlaced read;

- Up-the-ramp non-destructive sampling;

- Fowler non-destructive sampling.

Here it follows a brief description of the different read-out modes:

Reset-Read mode: this is the basic read mode, in which the detector is first reset (line by line) and then read-out. It is the fastest mode for full frame mode, but the noise associated to the reset value makes it not useful for science observations. In general, it is kept for diagnostics. 


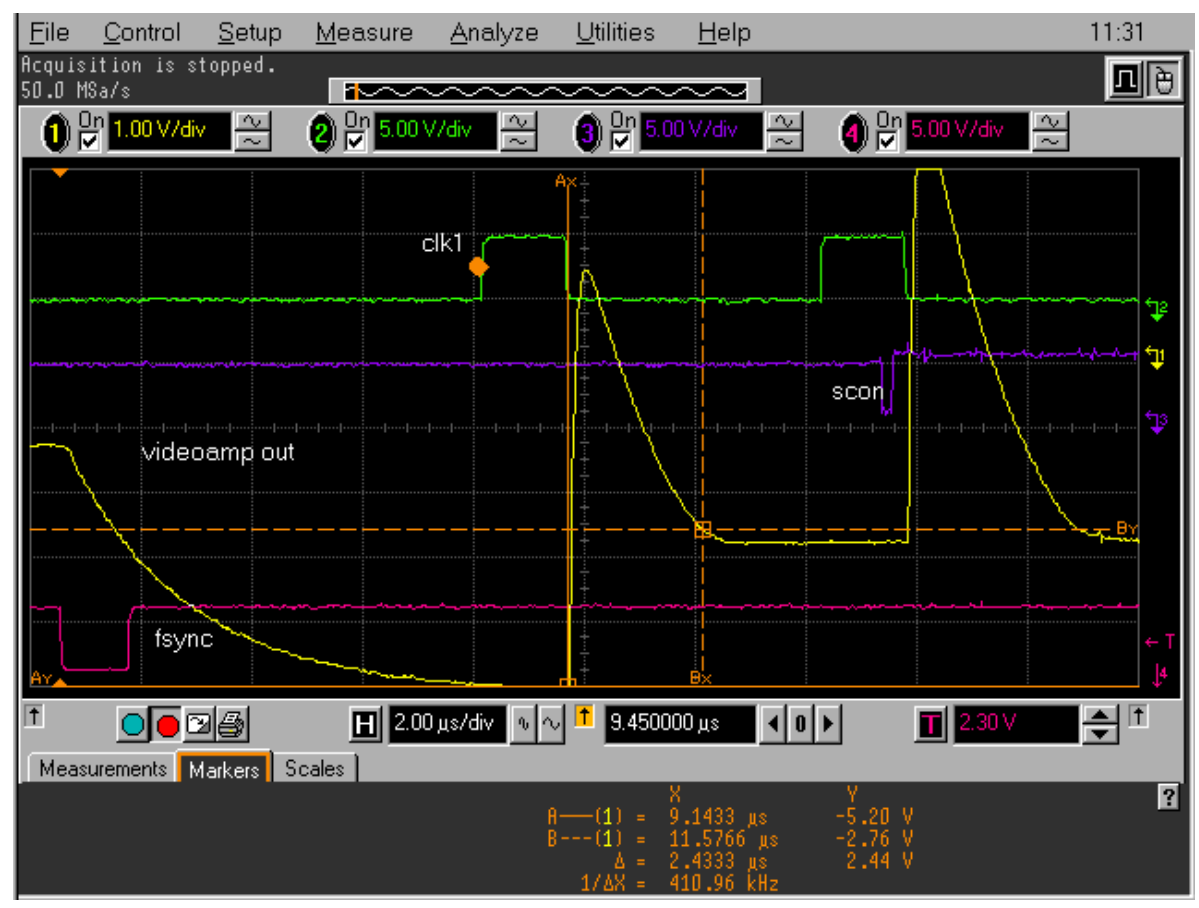

Figure 2. Scope shot of one output channel

Reset-Read-Read mode: this mode (Correlated double sampling) is used to eliminate the correlated noise due to variations in the reset voltage. The detector is reset like in RR mode, and it is then read out nondestructively. After the selected integration time, the chip is read-out again. Any reset anomaly and kTC noise in the reset is present in both read frames, and therefore in eliminated in the difference. This mode is the default in high background applications, where background limited performances are reached easily.

Line-interlaced mode: similar to the RRR mode, except that each line of the detector is read, then reset, and then read again. The signal is obtained by the difference between the first read (which samples the signal after the integration time) and the second read of the same line on the previous "frame". This mode is more time-efficient than the normal RRR mode.

Up-the-ramp sampling: in this mode, the detector is reset and then read-out non-destructively several times. The signal is computed alternatively as the average of all the frames (this would give the mean photon rate) or as the slope of the line signal versus time (more computing intensive). The read-noise is reduced roughly as the square root of the number of samples. More complicated data reduction can allow to reduce the impact of cosmic rays and other glitches.

Fowler sampling: another non-destructive read out scheme. A number of reads is performed after the reset, and the same number of reads is performed after the integration time. The signal is the difference of the average of the two samples. It also reduces the read noise by the square root of the number of reads. It is normally preferred to Up-the-ramp sampling but doesn't allow cosmic rays reduction (or at least not as efficiently).

In the context of Lucifer operations, where we expect in most modes a low background flux, the need for small read-out noise and at the same time for long integration times makes non-destructive readouts the preferred mode. 


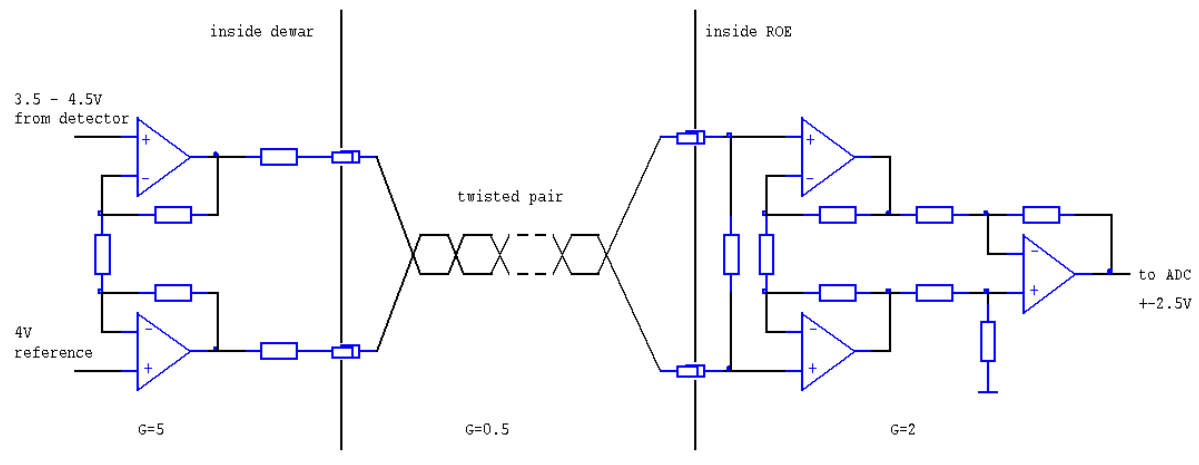

Figure 3. Scheme of signal chain

\subsubsection{Use of internal source followers}

A scheme of the internal circuitry is shown in Fig. yy. The Hawaii-2 detector possess an internal source follower at the outputs, which can be disabled at will. The main reason to disable the internal source follower is to reduce the glowing which is produced by these elements when are operated. This effect can be easily seen even after a few seconds of integration and locally dominates the counts coming from the dark current.

When using the internal source follower amplifier, all outputs require a pull-up resistor to properly bias the amplifier. Typically, RSC suggest to employ a $10 \mathrm{~K} \Omega$ resistor to 5 volts. Alternatively, an external current source can be used to bias the amplifier.

The operation of the output source followers is controlled via the DRAIN signal. The source follower output amplifier is enabled by making the DRAIN input 0 volts (buffered mode), while a DRAIN input voltage of $5 \mathrm{~V}$ bypasses the output amplifier (unbuffered mode). The preferred mode for Lucifer is the unbuffered mode. In this mode, the load resistor is removed from the read channels; otherwise, the additional current could cause an overflow in the pixel source follower. On the other hand, as can be seen in Fig. Reference output, the internal source follower of the reference channel cannot be switched off. We kept the load resistor in place, in this case, in order to be able to still use the reference channels in unbuffered mode. The usefulness of the reference channels in this mode has to be verified.

\section{READ-OUT ELECTRONICS}

The readout electronics consists of three main groups:

The Detector Frontend Electronics (DFE) generates the correct potentials for control of the detector and amplifies the output signals of the detector. The IR array detector is located inside the vacuum vessel on a fanout board which contains 32 preamplifiers for data output and four for reference signals. The clockdriverand biasboard is be placed outside the vessel. In Fig. xx one can see the Fanout board in the test dewar. The detector is looking down, and some of the amplifiers have a temperature sensor we used to determine the thermal behaviour of the board itself.

The Data Acquisition and Control Electronics (DACE) generates all control signals, digitize the output signals and transmits them to Computer Data Interface (CDI). A Control Processor Board (CPB-3) houses the digital signal processor (DSP) which controls all other Boards. The controlling and timing is freely programmable from the host computer. The selected readout-pattern is loaded from host computer by DSP into the Pattern Generator Board (PATGEN). The sequencer uses a quartz time base (min. $50 \mathrm{~ns}$ ). The detector output signals $(32+4$ channels) are amplified and simultaneously digitized by 16 Bit ADCs $(2 \mathrm{MHz})$ on the acquisition board (QADC + IRDIFAMP). The readout (16 bit word) of the ADC register and transfer of the data via fiberoptic link to the computer data interface (CDI) is controlled by BUSLINK board. Temperature are measured in the 


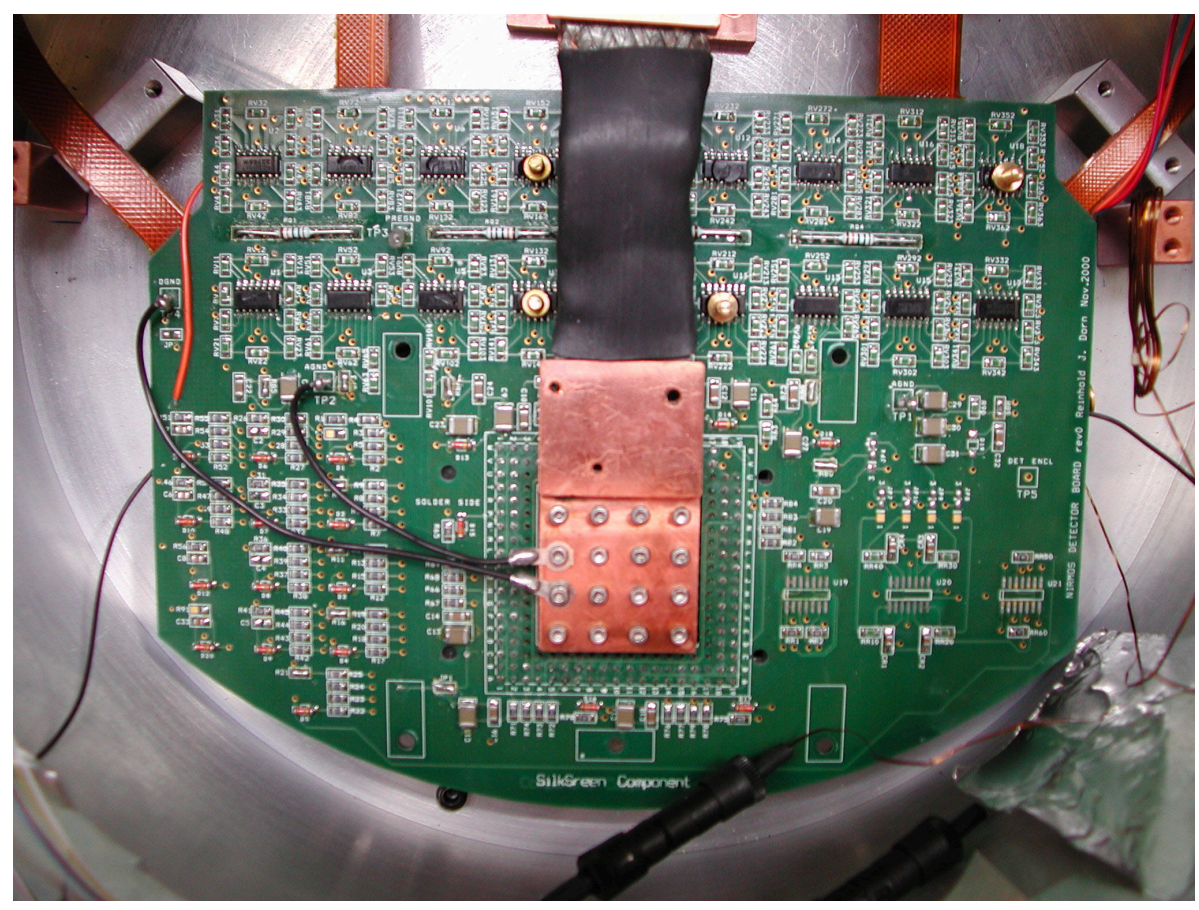

Figure 4. Fanout board

electronic box by ICB board. Backplanes connect the different boards and allow a simple and clear construction as well as a good EMC behaviour. The maximal cable length between the two electronics (DFE and DACE) is $10 \mathrm{~m}$.

The Computer Data Interface (CDI) is near the host computer (SUN). Two GLINK boards receive the serial data from the BUSLINK boards (in DACE) and convert them into parallel data. The data is FIFO buffered and transmitted to the I/O interface boards ( 2 x PCICD60 board, 2 x 60 Mbyte/sec).

All boxes, boards and cables are specifically designed and build according to the EMC criterion.

\subsection{Front end electronics}

\subsubsection{Clock board}

The clockboard receives the clocking pattern from the pattern generator board that is located in the data acquisition electronic box. There are 13 clock lines, which are galvanically isolated from the pattern generator by optocouplers. The optocouplers drive analog switches which apply the appropriate signal levels to the detector.

\subsubsection{Bias board}

The biasboard provides 14 adjustable low noise, low drift biases:

- detector power supply;

- detector reset voltage;

- power supply for the detector output source followers;

- biasgate and biaspower;

- $4 \mathrm{~V}$ reference for the fanoutboard.

All biases are derived from a single precision reference voltage. Every voltage is adjusted with a CERMET potentiometer and buffered with an opamp. All the biases are filtered with a ceramic capacitor. 


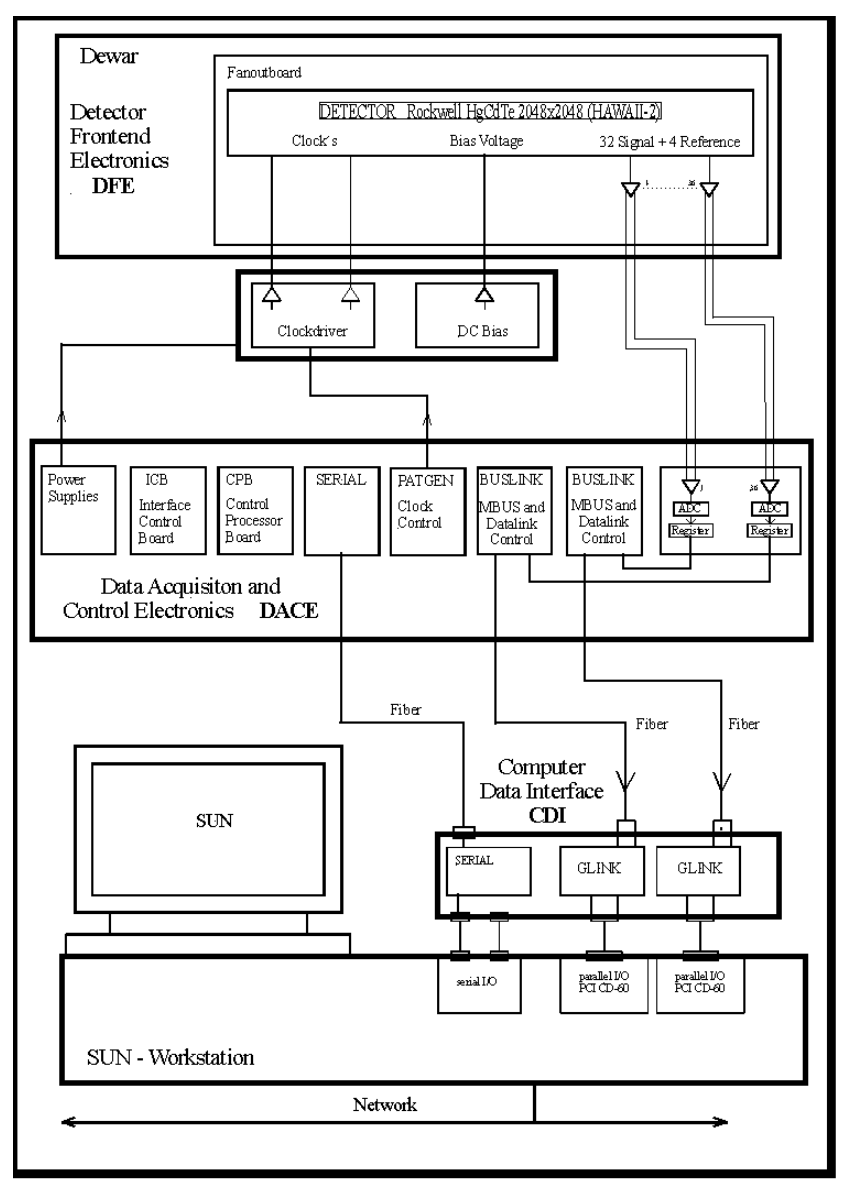

Figure 5. Block diagram of MPIA Read-out electronics

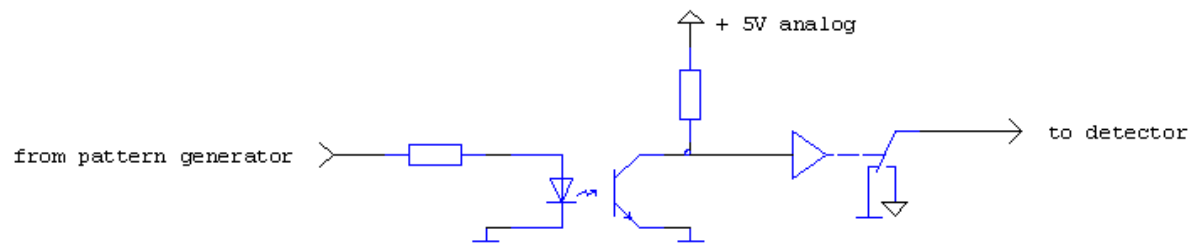

Figure 6. Scheme of the clock circuit

\subsubsection{Data acquisition and control electronics}

The data acquisition and control electronic box contains the components for generating the pattern, digitizing the video signal and sending the video data to the computer data interface. These are:

- Instrumentation Control Board;

- Control Processor Board;

- Pattern Generator;

- Buslink Board;

- Quad ADC Board; 


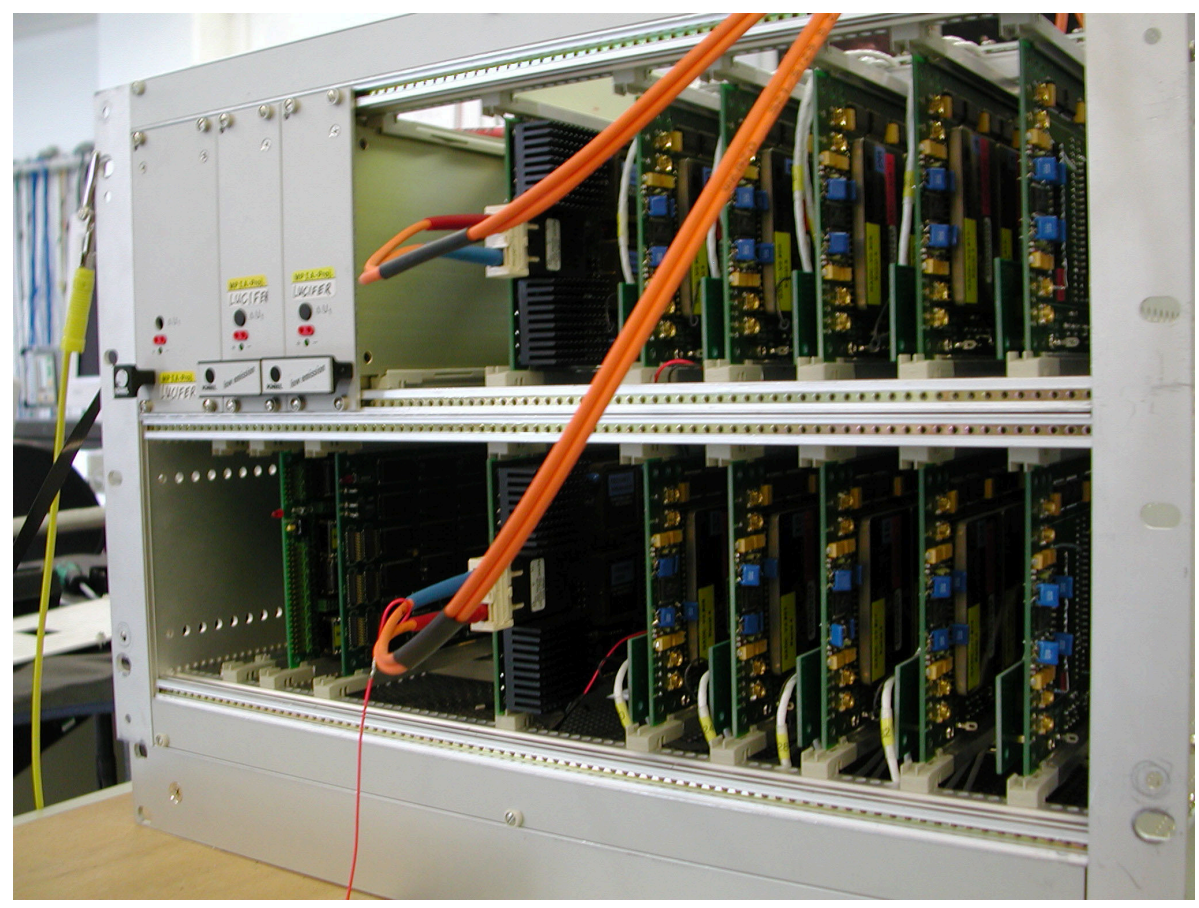

Figure 7. Control electronics

- Power Supply.

Instrumentation Control Board (ICB) The ICB contains several functions often needed for monitoring, recording and control of system parameters. The ICBs job in the LUCIFER ROE is to measure the temperature inside the case. All the other functions are not used at the moment.

The Control Processor Board (CPB) The CPB controls the activities of the whole ReadOut Electronics. This is done by means of a Texas Instruments Digital Signal Processor TMS 320C30. The board includes 256k x 32 bits of programmable memory (EPROM) and 256k x 32 bits of static RAM. After power-on, the program resident in the EPROM is loaded from there to the RAM and is executed there to achieve a faster execution. In addition to two serial interfaces supporting data rates up to $115 \mathrm{kBaud}$, the CPB is equipped with appropriate registers which provide a maximum of 64 bits parallel I/O. The serial interface is connected to a fiber transmitter module in order to avoid ground loops.

The Pattern Generator (PATGEN) The selected readout pattern is loaded via the serial line in the RAM of the CPB. The CPB transfers the desired patterns via the system bus to the pattern generator. Its important to notice that the PATGEN as well as the CPB can keep up to 256 different patterns. The ability of the PATGEN to sequence and loop different patterns in any combination gives the system a high flexibility and fits the need for clocking a wide range of CCDs and FPAs. A sequence of patterns is stored in a so called instruction table. A number of different instruction tables can be stored in the RAM of the CPB, one of them can be transferred to the pattern generator and executed. Refer to chapter 4 for additional information. There are two important bits in the pattern the start conversion bits. They connect to the Buslink board and thats the next topic.

The Buslink Board (BUSLINK) The buslink board does two jobs: it controls the handling of the ADCs and second puts the data from the ADCs to the fiberlink subsystem. If the buslink receives a start conversion 
pulse from the PATGEN it starts a programmable hardware state machine. This state machine waits until all ADCs have completed their conversion and after that generates the needed signals to read out the conversion results. Programmable state machine means that you can specify which ADC on which board has to be read out. This command is first sent to the CPB and goes via the system bus to the buslink control registers. The gathered data is now put in the fiberlink interface registers and transferred to the Computer Data Interface. The necessary timing is also generated by the programmable hardware state machine.

The differential line receiver (DLR) The DLR rejects any injected common mode noise and translates the differential signal from the fanout board to single ended. The IRDIFFAMP is mounted piggyback on the QADC and contains 4 channels. Refer to figure 2 for additional information.

The Quad ADC Board (QADC) The voltage coming from the IRDIFFAMP is digitized by $2 \mathrm{MHz} 16 \mathrm{bit}$ ADCs (Analogic ADC 4322A). Each board holds 4 ADCs. The 32 video outputs of the detector require 8 full equipped QADC boards. The 4 detector reference channels are split to two QADC boards with two ADCs each. Therefore the system can be split in two identical halves.

Power Supplies The DACE box contains the necessary power supplies for itself and the detector frontend electronic. These are:

- 1 switch mode supply $5 \mathrm{~V} / 16$ Amps for the digital circuits;

- 2 switch mode supplies $15 \mathrm{~V} / 4$ Amps for the analog circuits;

- 1 linear regulated supply +- 8V / 2Amps for the detector frontend electronics.

The Computer Data Interface (CDI) The CDI contains two GLINK boards and reconverts the optical signal from the fiberlink into 16 bits of parallel data and transmits them to the I/O connections of the workstation. The data transmission to the host computer is buffered with a FIFO to avoid overrun errors. The standard size of the FIFO is $128 \mathrm{k} * 16$ bit. With a piggyback board it can be expanded to total $512 \mathrm{k} * 16$ bit.

\section{TEST RESULTS}

In this section we discuss the results of the tests performed with the science detector for LUCIFER I. These tests were performed with the first prototype of the Read-out electronics, the NIRMOS fan-out board and a test dewar cooled with Liquid Nitrogen. Due to the restrictions of the test setup, some of the results are still provisional.

\subsection{Cooling of the Fan-out board}

The power dissipation of the operational amplifiers used in the NIRMOS fan-out board is quite high (2-2.5 W in total); this causes the temperature of the amplifiers to raise quickly up to $120 \mathrm{~K}$ and beyond. The output from the amplifier is independent on the temperature, but at low temperature there can be a shutoff of the amplifiers (spiega meglio). In any case, keeping the detector below $77 \mathrm{~K}$ as required for operation, and at the same time having the amplifiers on the fan-out board at $120 \mathrm{~K}$ poses big problem to the cryogenic concept. For this reason we are developing a modification of the fan-out board, in which the detector carrier is connected to the fan-out board only with a flexible cable and is cooled independently from the fan-out board, which is in addition shielded to avoid the glowing from the amplifiers to reach the detector. This new solution is not yet tested. 


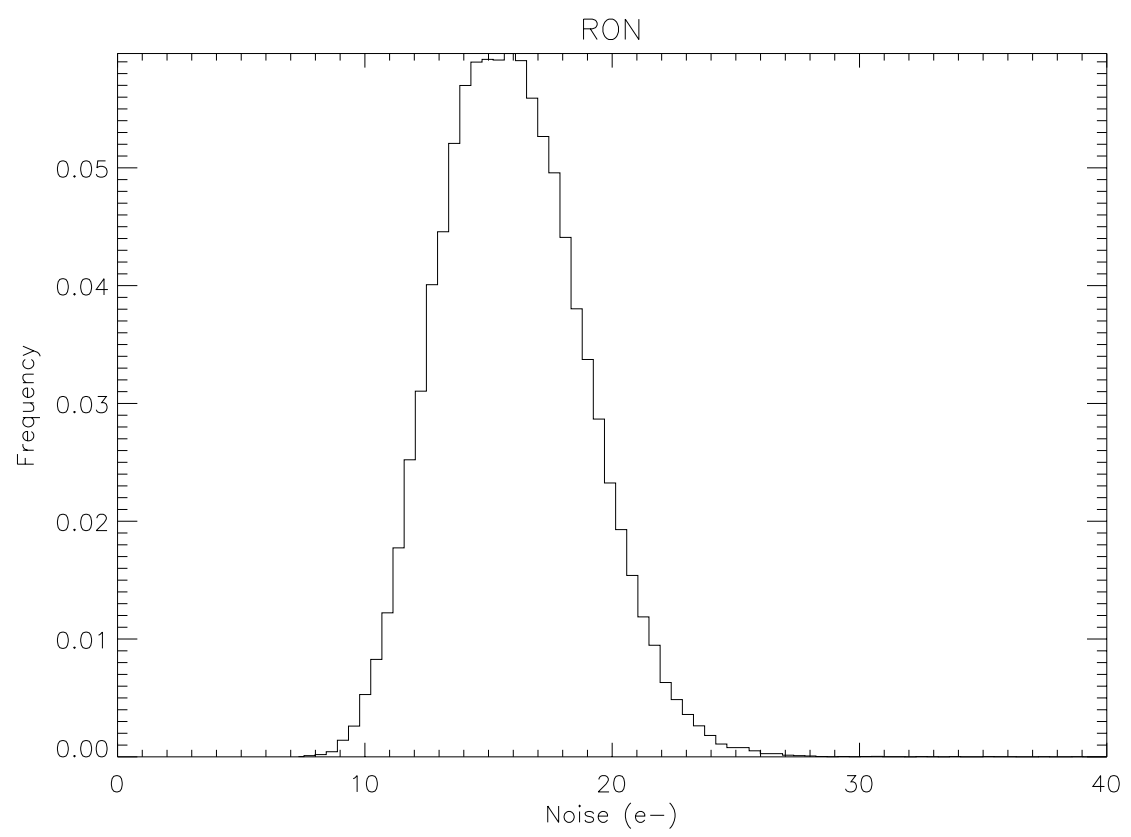

Figure 8. Histogram of noise values

\subsection{Read-out noise}

We have measured the read-out noise $(\mathrm{RON})$ of the Lucifer detector at different temperatures between 75 and $85 \mathrm{~K}$. We used two different methods: the RON is evaluated through the spatial noise measured in the difference between two consecutive frames taken with the shortest exposures time and in dark conditions. Using this method one must pay attention to the presence of noisy pixels, which will modify significantly the computed variance. A number of selected areas will be analyzed, and the final result is the average of the noise measured in different pairs of frames and on the various reference areas.

Using the second method, the noise is evaluated, pixel by pixel, as the variance around the mean of the pixel during a sequence of frames taken in the same conditions stated before. This method has the advantage of generating a noise map, where is straightforward to identify and mask noisy pixels. In addition, one can also look at the distribution of noise values, which sould be close to a poisson curve (?). In fact, one can use both methods on the same sequence of files, and should get basically the same answer.

The results, obtained with the second method, are shown below. The median value for the noise (in Correlated Double sampling mode) is about $15 \mathrm{e}^{-}$; this is consistent with a RON for each read of about $10 \mathrm{e}^{-}$. In Fig. xx is shown the noise distribution measured.

The system noise, that is the noise procuced by the whole analog and digital electronics with the detector outputs substituted by a constant low noise voltage, is below one count, which with the conversion factor of 4.5 means about 4.5 electrons. Such a system noise implies a contribution of about $10 \%$ to the total noise.

\subsubsection{Linearity and well depth}

The linearity has been verified to be better than $1 \%$ over a range of about $3.0 \cdot 10^{4}$ counts (aggiungi grafico e dettagli. The usable well depth is in excess of $3.5 \cdot 10^{4}$ counts, corresponding to $1.5 \cdot 10^{5}$ electrons.

\subsubsection{Noise reduction techniques}

In order to reduce the read-out noise, which is particularly important in order to meet the stringent requirements set by the operation with LUCIFER, we implemented (as described above) both Up-the-ramp and Fowler multiple sampling strategies. Since, as shown by paper di Finger e/o company, the effective noise scales as ....., it will be sufficient to have 8 Fowler pairs to reach a noise level of $5 e^{-}$. 


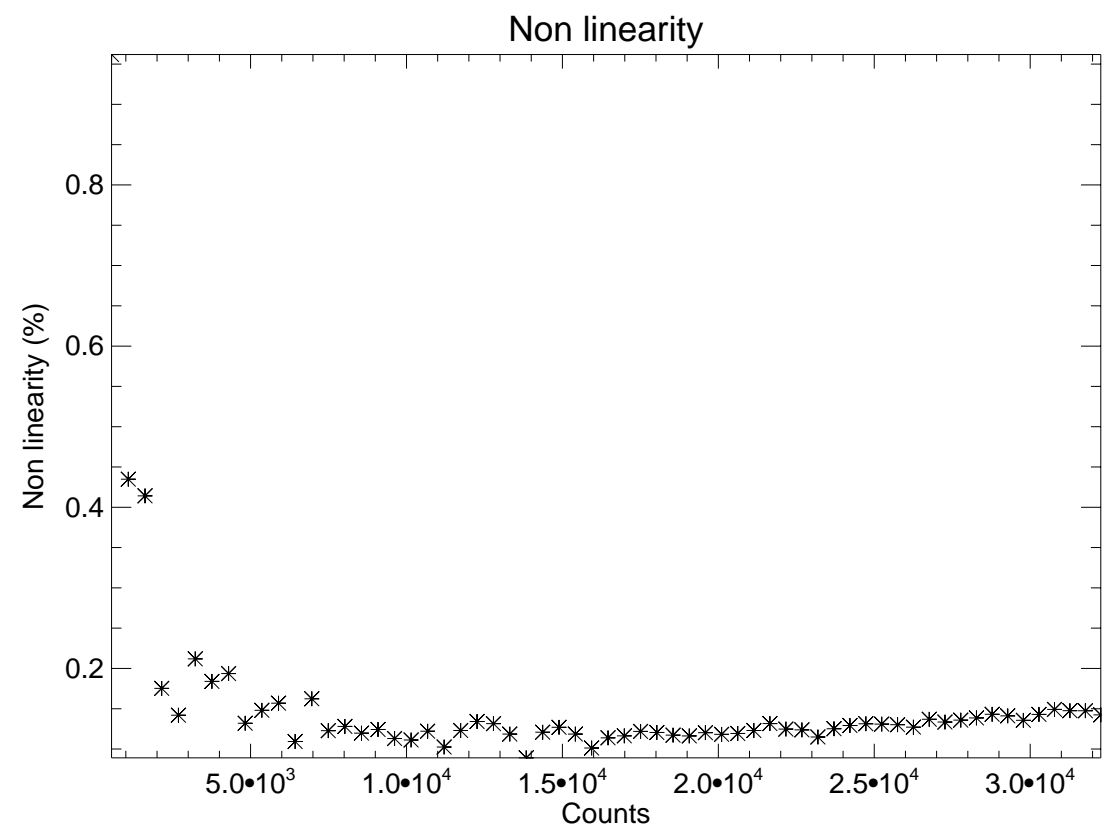

Figure 9. Non linearity

\subsubsection{Long term stability and reference channels}

In order to further improve the performances of the detector, we planned to use the reference channels available on the Hawaii-2 MUX. As described above, the Read-out electronics has been designed to accomodate these four additional output. Unfortunately, the reference channels are easily implemented only in the so-called buffered mode, in which the on-chip output source follower ampifiers. This mode is not desirable, though, because of the glowing of these amplifiers which introduces an additional source of dark current, especially at the edges of the field, which is not easily removed.

Once the unbuffered mode is used, in which the on-chip amplifiers are switched off, the glowing is largely removed, but (as shown by the diagram in Fig. xyz), it is then necessary to remove the load resistors at the outputs. Since in the reference channels the output source follower is never switched off, there is an additional current source which would bring the detector source follower out of range. We therefore decided to keep the load resistors only for the reference channels. By doing so, it is not clear whether the output from the reference channel can be used to monitor drifts on the science channels. This will be verified in the next test runs.

\section{ACKNOWLEDGMENTS}

Di nuovo cani e porci

\section{REFERENCES}

1. C.A.L. Bailer-Jones, P. Bizenberger, C. Storz, "Achieving a wide field near infrared camera for the Calar Alto 3.5m telescope", in Optical and IR Telescope Instrumentation and Detectors, Proc. SPIE 4008, p. 1305, 2000 .

2. S. Ligori, U. Graser, B. Grimm, and R. Klein, "Design and tests of the MIDI read-out electronics" in Interferometry in Optical Astronomy, Proc. SPIE 4006, p. 136, 2000. 